\title{
Les arcanes du sujet
}

Jean-Paul Desgoutte

\section{OpenEdition}

Journals

Édition électronique

URL : http://journals.openedition.org/communicationorganisation/2011

DOI : 10.4000/communicationorganisation.2011

ISSN : 1775-3546

\section{Éditeur}

Presses universitaires de Bordeaux

\section{Édition imprimée}

Date de publication : 1 novembre 1997

ISSN : 1168-5549

\section{Référence électronique}

Jean-Paul Desgoutte, "Les arcanes du sujet », Communication et organisation [En ligne], 12 | 1997, mis en ligne le 26 mars 2012, consulté le 20 avril 2019. URL : http://journals.openedition.org/ communicationorganisation/2011; DOI : 10.4000/communicationorganisation.2011

Ce document a été généré automatiquement le 20 avril 2019

(c) Presses universitaires de Bordeaux 


\title{
Les arcanes du sujet
}

\author{
Jean-Paul Desgoutte
}

1 Le sujet dont nous allons esquisser la figure, est le sujet du récit. Nous en aborderons l'étude par deux chemins distincts mais convergents : celui ouvert par la linguistique - en particulier dans son devenir pragmatique et narratologique -et celui qui se dessine peu à peu dans les recherches consacrées à la syntaxe narrative du film.

2 L'analyse du verbe et l'analyse de l'image ont développé des stratégies distinctes du fait qu'elles traitent d'objets dont la nature est différente. Le signe verbal est de nature symbolique ou digitale ; autrement dit, sa fonction sémiotique est liée à la médiation d'un code qui lève l'arbitraire attaché à son support signifiant. Le message visuel - l'image quant à lui, est de nature analogique ; autrement dit, la relation qu'entretient le signifiant visuel à l'objet de référence est nécessaire et immédiat.

3 La nécessité d'une convergence des deux approches, verbale et visuelle, est liée entre autres au développement des nouvelles technologies de numérisation et de compression, qui autorisent dorénavant la conception de récits hétérogènes - qui mêlent texte, parole, son et image - dans un univers énonciatif renouvelé par le jeu interactif, l'univers du multimédia ${ }^{1}$.

4 Le multimédia est né de la rencontre de deux technologies qui se popularisent parallèlement depuis vingt ans, l'informatique - technologie du texte et du code - nourrie entre autres à l'héritage de près d'un siècle de linguistique, et la vidéo - technologie de l'image et du son - nourrie quant à elle de l'héritage de cent ans de cinéma. Il manifeste donc l'intégration des deux traditions narratives du texte et de l'image, transformées par l'introduction du jeu interactif qui inscrit dorénavant le sujet et ses fluctuations comme objet intégrant de l'analyse du récit.

5 La question dont nous allons traiter pourrait donc se formuler de la façon suivante: «Comment rendre compte de la résonance subjective de ce qui s'énonce dans le récit?» 


\section{L'approche linguistique}

6 La linguistique saussurienne - ou linguistique du signe -a limité son objet d'étude à l'analyse des constituants de la proposition et porté son intérêt exclusif à l'énoncé délivré de son contexte - dans ce qu'il porte d'information structurelle sur le code qui le régit. Dans cette démarche, la langue est conçue comme le plus petit dénominateur commun aux membres de la communauté qui l'utilise. Le premier temps de la linguistique a donc eu pour préoccupation d'évacuer radicalement la question du sujet, afin d'accéder aux invariants du système verbal. Il s'agissait en fait de délivrer l'étude de tout ce qui du sens de l'énoncé peut être lié à la finalité de l'énonciation.

7 Cette précaution heuristique laissait de côté, aux psychologues, le soin de traiter de ce qui s'échange, au-delà de l'information sémiotique et par-delà le système grammatical.

Cette démarche, qui a permis de jeter les bases d'une analyse formelle du langage délivrée de l'héritage mentaliste, a donné lieu dans la deuxième moitié du siècle, à un rebondissement aux effets multiples. La description, par Benveniste d'un ensemble d'éléments du langage qui ne renvoient pas au code mais directement au contexte de leur énonciation, les déictiques, ouvre la brèche d'une réflexion sur la double fonction sémiotique et sémantique du langage et sur la nécessité corollaire d'élaborer, à côté de la linguistique du signe, une linguistique de la phrase ou linguistique de l'énonciation ${ }^{2}$. L'énoncé, qu'il serait dorénavant sans doute préférable d'appeler le message, mêle - selon les termes avancés par la philosophie analytique ${ }^{3}$ et repris par Benveniste ${ }^{4}$ - une fonction constative (ou dénotative) à une fonction performative (ou pragmatique). Ce qui s'échange dans la parole, c'est à la fois une information décryptable avec les outils du code et une intention sur l'autre. La parole n'est pas seulement un outil de représentation, c'est également une façon d'agir. C'est ainsi que la question du sujet a été réintroduite dans les études consacrées au langage, dès lors que la linguistique a cessé d'être exclusivement une science du code.

9 Les travaux européens de Benveniste, d'Austin et de Jakobson ${ }^{5}$, mettant en évidence la multiplicité des fonctions à l'œuvre dans l'usage de la langue, élargissent le champ de la recherche linguistique à l'étude de l'énonciation du discours et du récit, et jettent également un pont vers les théories de la communication qui se développent aux EtatsUnis.

10 La réinscription de l'énoncé dans son contexte, oblige de s'interroger à nouveau sur la nature de l'axiome que constitue la proposition linguistique. Il s'agit dorénavant d'explorer l'au-delà de la phrase à la fois dans sa manifestation discursive - la conversation ou le dialogue - et dans sa dimension narrative. En chaque cas en effet on a affaire à un enchaînement de propositions dont la signification globale outrepasse manifestement la somme des significations particulières.

11 La ponctuation intersubjective du discours et la syntaxe narrative du récit deviennent les lieux privilégiés d'observation de ce qui se joue entre les partenaires de l'échange verbal et donc du rapport entre le sujet et la langue. 


\section{Le récit filmique}

12 La définition la plus simple du plan visuel est celle qui consiste à le décrire comme l'ensemble des images enregistrées entre le moment où on met en route le moteur de la caméra et le moment où on l'arrête. Autrement dit le plan restitue ou simule la continuité spatiale et temporelle d'un regard. Cette définition nous offre d'emblée la méthode et les outils nécessaires à l'analyse du film, tout en nous apportant des informations essentielles sur la nature et l'enjeu du récit. Elle met en effet en évidence les trois éléments qui caractérisent les segments narratifs: la subjectivité, la temporalité et l'espace. Le récit nait d'une fragmentation et d'une recomposition du rapport que le sujet entretient au temps, à l'espace et à l'autre.

13 Le cinéma met donc en évidence de façon spectaculaire la nature discursive de la fragmentation du récit. L'enchaînement de propositions qui caractérise tout récit procède de la double nécessité de rassembler en une même chaîne symbolique l'alternance des points de vue et l'alternance des instances. Cette nécessité est liée à la fonction centrale de tout récit, voire de tout langage, qui est de créer le lieu d'une rencontre intersubjective d'univers et de points de vue radicalement disjoints.

Le segment visuel donne à voir un contenu, représentant analogique de l'événement, tout en définissant un hors champ spatial et un hors champ temporel, dont les coordonnées sont implicites. Il manifeste également un sujet du regard qui appartient soit à l'univers référentiel attaché au contenu de l'image, soit à l'univers énonciatif qu'il présuppose en tant que message. L'enchaînement de plans qui constitue le film peut ainsi être assimilé à une concaténation de regards que l'on peut classer en deux catégories :

- les regards dont le point de vue est interne à l'événement représenté, qu'on qualifiera de topiques ${ }^{6}$. Ces regards appartiennent à la diégèse ${ }^{7}$, ou encore à l'« histoire ».

16 - les regards dont le point de vue est externe à l'événement représenté, qu'on qualifiera d'utopiques. Ces regards appartiennent au discours ou à l'énonciation.

17 Dans cette perspective, la rupture de continuité du récit filmique - la coupe ou le raccord, qui sont le privilège de l'énonciateur - manifeste soit un changement d'instance (topique / utopique), soit un changement de point de vue à l'intérieur de l'instance. La coupe institue un double paradigme de segments topiques et utopiques dont l'enchaînement ou la distribution manifeste la mainmise énonciative sur l'exposé de l'événement.

\section{Le point de vue}

Le regard en creux que révèle toute image peut lui-même être objectivé. C'est l'effet miroir ou contrechamp. Si l'on appelle point de vue le plan de l'objectif et plan d'application le plan sur lequel se fait la mise au point, ces deux plans sont l'un par rapport à l'autre dans une situation de champ/contrechamp. Toute image porte en elle implicitement son contrechamp. Le point de vue implicite de toute image renvoie soit au regard d'un acteur de l'événement (point de vue topique), soit au regard du narrateur (point de vue utopique), qui relaie en tous cas le regard du spectateur.

19 Mais l'image en contrechamp ne peut pas être contemporaine du regard qui la révèle: toute nouvelle image implique en effet un nouveau regard ou encore l'intervention d'un narrateur, témoin «hors cadre» de ce qui se joue entre le regard et l'objet. Cette 
dialectique des regards est tout à fait analogue à la dialectique des personnes grammaticales telle qu'elle a été décrite par Benveniste ${ }^{8}$. Je et Tu s'impliquent l'un l'autre et s'opposent ensemble à la troisième personne ou non-personne, Il. Le regard du tiers en ce cas, celui du narrateur - est en dehors du cadre où se joue l'échange. Il se propose cependant lui-même comme un message adressé au spectateur dans un nouveau jeu intersubjectif duel. C'est ainsi que le jeu des images et des regards anticipe ou accompagne le jeu de la parole et que l'un et l'autre peuvent être ramenés à un même champ d'analyse.

Le récit littéraire comme le récit filmique simulent la ponctuation des points de vue et des paroles qui constituent l'ossature même de tout échange. Ils en proposent un lieu de représentation homogène où s'inscrivent à la fois le jeu intersubjectif forclos qui lie les acteurs de l'événement et le jeu intersubjectif virtuel que le narrateur propose au lecteur.

21 Le récit est, selon l'expression d'Umberto $\mathrm{Eco}^{9}$, un bois qui se propose aux cheminements innombrables des lecteurs en promenade. On peut poursuivre la métaphore en affirmant que chaque rupture de point de vue dans le récit s'offre au lecteur comme un carrefour au promeneur. Elle oblige en effet le lecteur à lever l'implicite propre au hors champ spatial et temporel qu'elle induit de part et d'autre des segments narratifs concernés. Le choix du chemin à venir procède à la fois de l'interprétation des signes rencontrés précédemment et de l'anticipation de ce qu'évoquent les parcours disponibles.

Dans le dialogue ordinaire, la prise de parole procède d'une dynamique tout à fait comparable. Elle manifeste un changement de point de vue - de locuteur - et une continuité du propos qui exige que soit interprétés les ambiguïtés, les connotations et les implications corollaires à ce qui vient d'être dit, en fonction du projet illocutoire propre au nouvel interlocuteur. L'évolution du dialogue impose que les interlocuteurs valident peu à peu un certain nombre de propositions dont la somme constitue le sens de l'échange qui les rassemble. Cet ensemble de propositions partagées peut être considéré comme le récit de leur rencontre, en ce sens qu'il constitue désormais un fonds commun objectif qui prend valeur de contenu ou d'information. Nul doute cependant qu'en plus de ce fonds commun avéré, chacun des interlocuteurs retienne de l'échange un certain nombre de conclusions personnelles, ou subjectives, dont ils ne pourront faire le même usage.

On voit dès lors que chacune des propositions possède un contenu avéré et une série de contenus latents, qui demandent à être validés ou invalidés. Cette validation peut se faire selon trois modes ${ }^{10}$, un mode constatif qui manifeste l'accord des partenaires; un mode que nous qualifierons d'expressif qui correspond à l'interprétation que chaque partenaire fait pour son propre compte, en fonction de sa "culture» ou de la finalité de son engagement, et un mode que nous qualifierons d'impressif qui correspond à l'interprétation que chaque partenaire suppose être celle de son interlocuteur.

Le récit réserve au narrateur le soin de caractériser à son gré chacune des propositions émises, et ce à l'usage du lecteur qui se trouve pris lui-même dans un jeu intersubjectif de deuxième niveau où il est tenu d'interpréter l'enchaînement des ruptures et des raccords.

Le processus qui vient d'être décrit est un processus très général qui s'applique aussi bien au récit verbal qu'au récit filmique. L'image en effet peut être considérée comme une proposition - un message - dont la fonction est triple : constative, elle apporte un certain nombre d'informations sur le référent, expressive, elle manifeste le sujet ou le point de vue, impressive, elle révèle l'intenté du sujet sur l'objet du regard. 
On en vient donc à l'idée que tout récit, filmique ou verbal, peut donner lieu à une représentation multidimensionnelle, comparable à une partition musicale, qui révèle à chaque instant l'enchaînement des points de vue, topiques ou utopiques, selon trois registres, expressif, impressif ou constatif, rapportés respectivement à chacun des partenaires du jeu intersubjectif propre à l'événement de référence ou du jeu intersubjectif propre à la narration en cours.

\section{Le sujet}

Le sujet humain n'entre dans la communauté du langage que parce qu'il y est appelé. Il est Tu avant d'être Je. Et il n'est Je que par rapport à un Tu. D'où la difficulté à le décrire autrement que comme un processus ou une virtualité. En fait le mouvement propre au langage se fait en trois temps et deux mouvements, comme la valse. Un temps d'amour ou de désir -, un mouvement vers l'autre, un temps de silence, un mouvement de l'autre, un temps d'arrêt et de reconnaissance. Ce n'est que dans le troisième temps que les deux se retrouvent, hors cadre!

On peut encore le décrire à partir d'une métaphore visuelle. Le regard qui manifeste à la fois un point de vue et un objet, révèle un champ qu'il choisit et qu'il interpelle. Il le sollicite à l'exclusion du hors champ, mais n'achève son projet que dans la réponse, qui le révèle en contrechamp. Le sujet, sujet du langage, sujet de l'histoire n'apparaît qu'à la fin du processus, quand le cercle se referme et qu'un nouveau temps s'ouvre, porteur d'une mémoire où s'inscrit à la fois le champ et le contrechamp. La somme de ces deux images permet de reconstituer l'ensemble de l'espace au prix redoutable d'un temps de silence ou d'absence, le temps du raccord, qui introduit le temps dans l'espace et manifeste le fait que le regard ne peut jamais être contemporain de l'objet qu'il saisit, ou mieux encore que la réponse ne peut être contemporaine de la question. Le sujet qui reçoit la réponse n'est jamais exactement le même que celui qui l'a posée.

Le désir d'une présence absolue à l'autre ou à soi-même, d'un espace clos où le sujet et l'objet, l'un et l'autre, le je et le tu seraient enfin rassemblés dans un même instant, hante le cinéma depuis son origine (et la peinture depuis bien plus longtemps... ${ }^{11}$ ). C'est l'objet de tous les films qui mettent en scène leur propre énonciation, révélant la caméra pour faire croire naïvement que le sujet peut-être circonscrit. Le troisième temps du regard, en effet, rassemble l'un et l'autre dans le souvenir de leur relation commune. C'est le troisième temps du dialogue, ou temps du récit.

\section{Stade du miroir et stade de l'écho}

Il y a deux approches classiques - on pourrait dire quotidiennes - du sujet, une approche par le nom et une approche par l'image. L'identité, c'est à la fois le nom et l'image, le baptême et le portrait, l'interpellation et la photographie.

31 On voit bien, dès à présent, qu'en chaque cas le sujet reçoit son identité de l'extérieur, d'un autre qui le nomme ou le décrit, et que c'est à partir de cette profération, de cet acte de parole - même s'il s'agit d'image -, que se constitue le noyau autour duquel se développera - comme la perle autour du grain de silice - l'enveloppe feuilletée des innombrables identifications à venir. 

souvenir que l'un et l'autre élaborent de leur rencontre. C'est dire que je ne suis pas le produit de mes actes mais bien le produit d'un désir négocié avec ceux dont j'accepte le regard. Le sujet se développe par la médiation de l'autre. Et s'il peut développer des stratégies amoureuses ou autres qui lui autorisent l'espoir de quelque retour, il n'en est en tous cas jamais le maître. Le langage, bien commun par excellence, semble avoir inventé cette façon radicale de privilégier, une fois pour toutes, le devenir collectif à la réussite individuelle.

Si l'on veut bien admettre donc, que le sujet naît d'une scission de l'autre, on cherchera à en rapporter toute description à une complémentarité implicite. On s'interrogera sur les figures nouées avec l'autre, qu'il s'agisse de figures linguistiques, ou de figures visuelles. Comment décrire en effet une danse en s'intéressant au seul mouvement de l'un des partenaires... (sans même parler de ces figures plus intimes auxquelles peut conduire un sentiment amoureux)? 


\section{Figures sémantiques} les choix qu'il propose à son lecteur, choix virtuels qui avant même d'être posés rétroagissent sur le travail de conception. L'intention ou la tension qui se créent entre les partenaires de tout échange devient donc un paramètre du récit. Le virtualité propre à un récit multimédia offre au lecteur un grand nombre d'interprétations, qui n'est pas infini, mais dont chacune des manifestations peut être relativement indépendante de l'intention explicite du concepteur. Le multimédia simule le choix du lecteur, il propose une surdétermination au cheminement. Les parcours virtuels, potentiellement innombrables, donnent à l'objet une complexité dont on peut penser qu'elle est analogue à la complexité même du sujet à la fois surdéterminé et libre dans ses choix. C'est dans la répétition des 
choix que le lecteur va se structurer en sujet. Le cheminement est imprévisible, il échappe au concepteur et peut le surprendre, même si ce dernier reste maître du jeu.

L'univers sémantique du récit interactif reste potentiel dans chacun des cheminements, les choix écartés participant à la signification de ce qui s'énonce, à la façon dont les harmoniques accompagnent la mélodie. Le multimédia déploie et concrétise le jeu harmonique propre à la signification, dans l'enchaînement des choix associatifs ou paradigmatiques qui constituent la chaîne signifiante.

Il y a donc là une simulation formelle de ce qui se joue dans la communication intersubjective banale, verbale ou non verbale, où chacune des propositions de l'un peut être interprétée de façon variée par l'autre en fonction de ce qu'on pourrait appeler sa grille de lecture ou son champ sémantique propre.

A mi-chemin entre discours et récit, le multimédia se propose dorénavant comme le lieu privilégié d'une analyse de la métamorphose du pragmatique en sémiotique, du relationnel en lexical, qui caractérise la production de sens propre au jeu intersubjectif.

\section{NOTES}

1. C'est dans ce champ de recherche que s'est constitué, à l'université de Paris S, ['Atelier de recherche intermedia qui s'attache à formaliser les processus interactifs a l'œuvre dans les nouvelles formes de récits, selon une triple approche linguistique, sémiologique et psychosociologique, rapportée a la pratique de la conception et de la production multimédia.

2. Voir à ce propos Emile Benveniste, «L'appareil formel de renonciation »et «La forme et le sens dans le langage » Problèmes de linguistique générale, tome II, Paris, Gallimard, 1967.

3. Voir à ce propos John L Austin, Quand dire, c'est faire, Paris, Seuil, 1970.

4. Op. cit. Tome I, chapitre XXII, Paris, Gallimard, 1966.

5. Roman Jakobson, Essais de linguistique générale, Paris, Minuit, 1963

6. Sur l'opposition topique / utopique, voir Jean-Paul Desgoutte, op. cit.

7. Sur le concept de diégèse, ibidem, chapitre IV

8. Op. cit. Tome I, «Structure des relations de personnes dans le verbe "

9. Umberto Eco, Su promenades dans les bois du roman et d'ailleurs, Paris, Grasset, 1996.

10. Voir Desgoutte. op. ci chapitre trois.

11. Lire à ce sujet l'analyse de Michel Foucault du tableau de Velasquez, Las Meninas, in Les mots et les choses. Paris, Gallimard, 1966).

12. Jacques Lacan. «Le stade du miroir comme fondateur de la fonction du Je », Ecrits, Paris, Seuil, 1966. 


\section{AUTEUR}

\section{JEAN-PAUL DESGOUTTE}

Université de Paris 8, Infocom Atelier de recherche intermédia 